\title{
Thromboembolic complications of COVID-19
}

\author{
Leonora W. Mui ${ }^{1} \cdot$ Joe F. Lau ${ }^{2} \cdot$ Hwayoung K. Lee ${ }^{3}$
}

Received: 26 May 2020 / Accepted: 29 October 2020 / Published online: 7 November 2020

(C) American Society of Emergency Radiology 2020

\begin{abstract}
The symptomology of patients afflicted with novel 2019 coronavirus disease (SARS-CoV-2 or COVID-19) has varied greatly, ranging from the asymptomatic state to debilitating hypoxemic respiratory failure caused by severe atypical viral pneumonia. Patients may also develop a hyper-inflammatory state that can lead to multi-organ failure. It has become increasingly apparent that, as part of the hyper-inflammatory state, COVID-19 infection increases susceptibility to systemic thromboembolic complications that can contribute to rapid clinical deterioration or demise. This article aims to review imaging features of various systemic thrombotic complications in six patients with moderate to severe disease. This case series includes examples of pulmonary embolism, stroke, right ventricular thrombosis, renal vein thrombosis, and aortic thrombosis with leg ischemia.
\end{abstract}

Keywords Computed tomography (CT) · Coronavirus · COVID-19 · Thrombosis · Thromboembolism · Pulmonary embolism

\section{Introduction}

Although COVID-19 was initially regarded as a pulmonary illness causing significant morbidity and mortality in the elderly and in those with underlying medical conditions, experience in New York - an early epicenter of COVID-19 in the USA - found that a significant percentage of younger patients less than 50 presented with moderate to severe symptoms requiring hospitalizations [1]. This case series highlights the thromboembolic complications affecting younger patients less than age 65 , including pulmonary embolism, stroke, right ventricular thrombosis, renal vein thrombosis, and aortic thrombosis with leg ischemia. The patients reported here had

Leonora W. Mui

LMui@northwell.edu

Joe F. Lau

JLau@northwell.edu

Hwayoung K. Lee

HLee13@northwell.edu

1 Department of Radiology, North Shore University Hospital, Zucker School of Medicine at Hofstra/Northwell, Manhasset, NY, USA

2 Vascular Medicine Program, Department of Cardiology, Long Island Jewish Medical Center, Northwell Health and Zucker School of Medicine at Hofstra/Northwell, Manhasset, NY, USA

3 Department of Radiology, Zucker School of Medicine at Hofstra/ Northwell, Manhasset, NY, USA initially presented in April 2020 to the emergency departments of several teaching hospitals located within the same New York-based health system. These cases were retrospectively identified by search of saved case lists. Inclusion criteria required age less than 65. Cases were confirmed with SARSCoV-2 polymerase chain reaction (PCR) laboratory testing. Written consent was obtained from all involved patients or families.

\section{Case 1. Pulmonary embolism and aortic thrombus}

Case 1 is a 46-year-old man without significant past medical history who was diagnosed with COVID-19 infection at an urgent care center six days prior to ER presentation. He had 2 weeks of progressive symptoms including high-grade fevers, cough, and generalized weakness, culminating with hypoxemic respiratory distress with an oxygen saturation of $70 \%$ on room air on day of ER presentation. Chest $\mathrm{CT}$ angiography demonstrated extensive bilateral peripheral and lower lobe ground-glass opacities typical of COVID-19 pneumonia (Fig. 1a), bilateral interlobar pulmonary arterial emboli extending to bilateral upper and lower lobe segmental and subsegmental pulmonary arteries (Fig. 1b), and aortic arch thrombosis (Fig. $1 \mathrm{c}$ and d). Parenteral anticoagulation with heparin was initiated upon diagnosis. Although his respiratory symptoms improved initially, the patient developed 
Fig.1 A 46-year-old man who presented with fever, cough, and shortness of breath. a Axial image shows bilateral peripheral ground-glass opacities (white arrows) commonly seen in COVID19 pneumonia. b Axial image shows numerous bilateral pulmonary emboli, including left lower lobe segmental pulmonary embolism. $\mathbf{c}$ and $\mathbf{d}$ Axial and sagittal images show focal partially adherent aortic arch thrombus (white arrow)
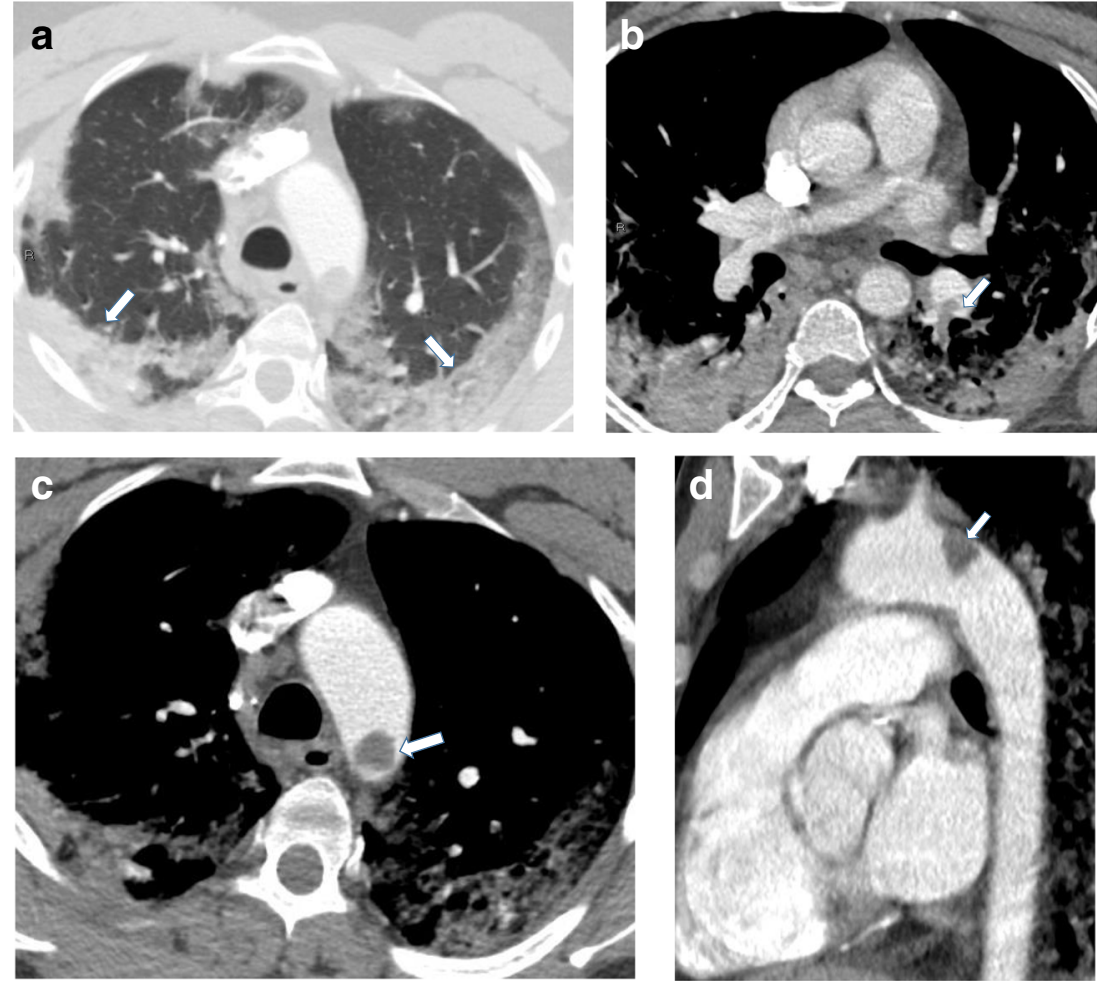

hypoxemic respiratory failure requiring mechanical ventilation on hospital day 7. With persistent respiratory distress and symptoms of systemic sepsis, an intravenous antibiotic (cefepime) was initiated for possible bacterial superinfection. On hospital day 10, he developed asystole with brief recovery of pulse, then returned to asystole, and died.

\section{Case 2. Cerebrovascular accident (CVA)}

Case 2 is a 40-year-old-man with essential hypertension and type 2 diabetes mellitus who presented with agitation and acute encephalopathy. Seven days prior to his current ED presentation, he was admitted with hypoxemic respiratory distress related to COVID-19. After initially stabilizing, he was discharged home without the need for supplemental oxygen. However, his condition worsened considerably, and he was readmitted with agitation, acute mental status changes, visual disturbance, and right-sided weakness. On physical examination for his second ED presentation, the patient was noted to have relatively preserved motor function but was aphasic and apraxic. Head CT showed multiple infarcts in the left frontal, bilateral temporal, and parietal lobes (Fig. 2a), with foci of petechial hemorrhage in the right parietal lobe. Subsequent neck CTA demonstrated multiple sites of thrombi distributed in various arterial sites, including the bilateral distal common carotid arteries, with extension to both carotid bulbs, the right internal carotid artery, and bilateral external carotid arteries (Fig. 2b). Head CTA showed occlusion of the right internal carotid artery (Fig. 2c) with distal reconstitution via collateral circulation as well as multiple bilateral M3 segmental occlusion (Fig. 2d). The imaging findings were consistent with thromboembolic stroke, likely owing to COVID-related hypercoagulable state. On hospital day 6 , the patient was started on intravenous heparin and monitored with daily head CT for potential worsening of petechial hemorrhage. During the second week of admission, the left MCA infarct was complicated by worsening intracranial hemorrhage. The patient was switched to enoxaparin to prevent further thromboembolic strokes. Due to dysphagia, a feeding gastrostomy tube was placed. The patient could speak but had difficulty comprehending speech. The patient was still hospitalized in his fifth week due to encephalopathy with plan to discharge home with home care. Follow-up phone call with the patient's spouse at 4 months after initial illness revealed that the patient still had severe difficulties with speech, verbal comprehension, self-feeding, and continence. He was reliant on his wife for all activities of daily living.

\section{Case 3. Right ventricular thrombus}

Case 3 is a 62-year-old man with essential hypertension, hyperlipidemia, and HIV with undetectable viral load (CD4 count of 800), presented with 1 week of worsening shortness of breath, cough, fever, and poor appetite. He was found to be hypoxemic with oxygen saturation of $77 \%$ on room air, along with COVID positivity via PCR testing. Admission chest 
Fig. 2 A 40-year-old man with hypertension and type 2 diabetes mellitus who presented with agitation, violent outburst, and aphasia and underwent head and neck CTA. a Axial non-contrast head CT image shows bilateral temporal infarcts (white arrows) with areas of petechial hemorrhage (star) as well as infarcts in the left frontal and parietal lobes (not shown). b Coronal maximal intensity projection (MIP) image demonstrates thrombi in bilateral distal common carotid arteries extending to carotid bulbs (white arrows), right internal carotid artery, and bilateral external carotid arteries (thin arrows). c Axial CTA image shows occlusion of the right internal carotid artery (white arrow). d RAPID image reveals an abrupt reduction in blood flow in the left M3 segment (white arrow)
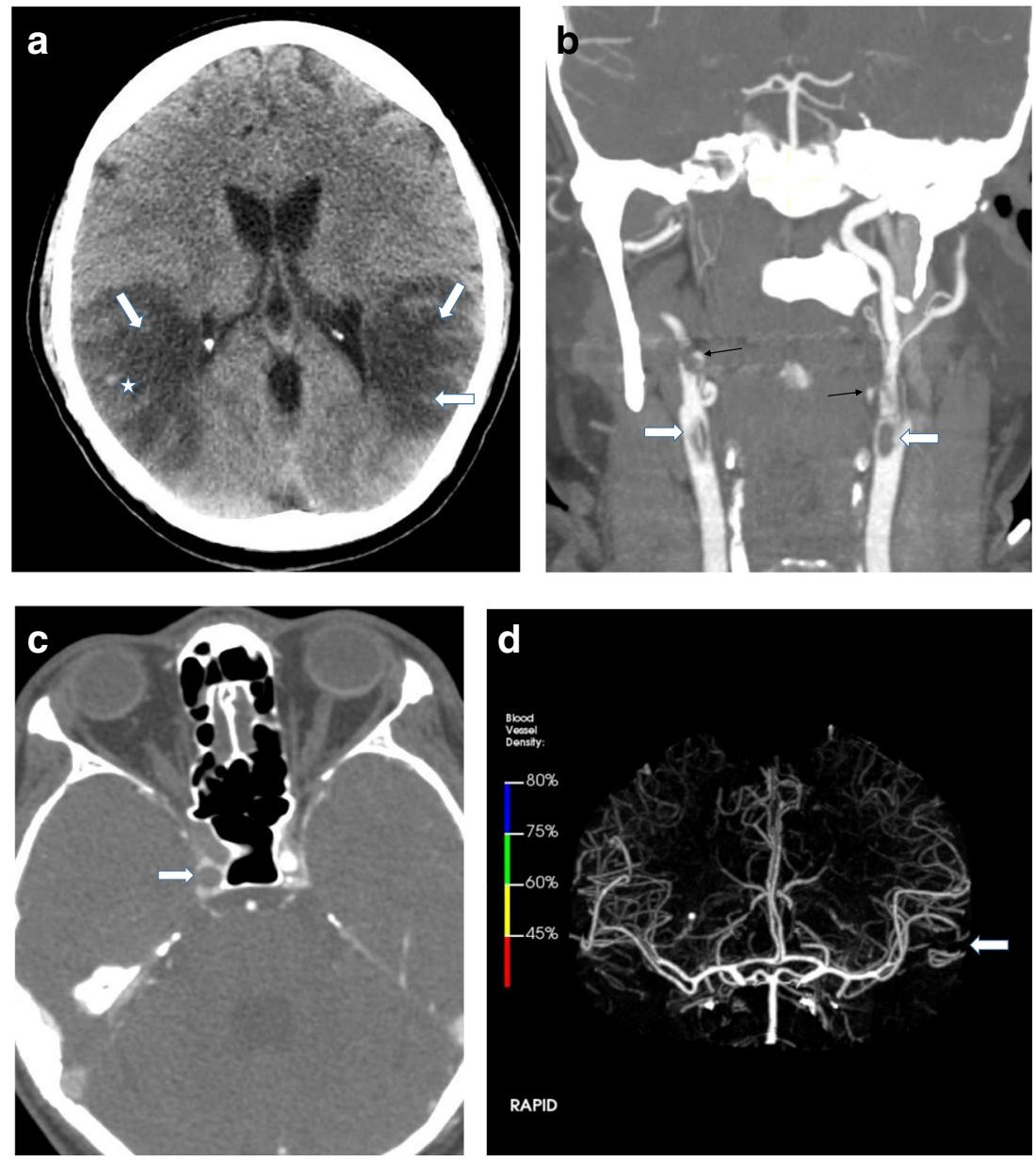

radiograph revealed moderate bilateral patchy infiltrates. On hospital day 5 , because of persistent hypoxemia despite receiving supplemental oxygen via non-rebreather, chest CTA was performed which revealed extensive ground-glass and patchy airspace opacities typical of COVID-19 pneumonia, bilateral lower lobe segmental pulmonary emboli, and right ventricular thrombus (Fig. 3). Echocardiogram subsequently confirmed the presence of a right ventricular thrombus. Because of this "clot in transit" with high probability of impending or concomitant pulmonary embolism, with concern for associated hemodynamic compromise, thrombolysis with t-PA was given, and the patient was subsequently maintained on parenteral heparin. He was discharged home on hospital day 15 with oral anticoagulation (apixaban) and home oxygen supplementation.

\section{Case 4. Renal vein thrombosis}

Case 4 is a 54 -year-old man with hyperlipidemia who presented with 3 days of right flank pain. He was started on an oral antibiotic for presumed pneumonia with associated fevers and cough 3 days before presentation to the ED. He subsequently tested positive for COVID-19. Urinalysis did not suggest the presence of a urinary tract infection. CT chest revealed right lower lobe pulmonary embolism (Fig. 4a). In addition, thrombosis was noted within the posterior tributaries of the right renal vein (Fig. 4b) with associated focal renal infarction. The patient was placed on anticoagulation (heparin first, then enoxaparin) and discharged home on apixaban 3 days later.

\section{Case 5. Peripheral arterial thromboembolic complications}

Case 5 is a 50 -year-old man with essential hypertension and hyperlipidemia who was initially admitted to a community hospital with 2 weeks of progressive dyspnea, fever, cough, nausea, and diarrhea. He was diagnosed with COVID-19 and discharged home with recommendations to self-isolate. Because of worsening hypoxemic respiratory distress, the patient was readmitted. On hospital day 6, he was transferred to ICU for hypoxic respiratory failure despite supplemental oxygen therapy via nonrebreather and after having already received medical treatment with steroids and hydroxychloroquine. On hospital day 10 , he developed sudden-onset left upper and lower extremity 


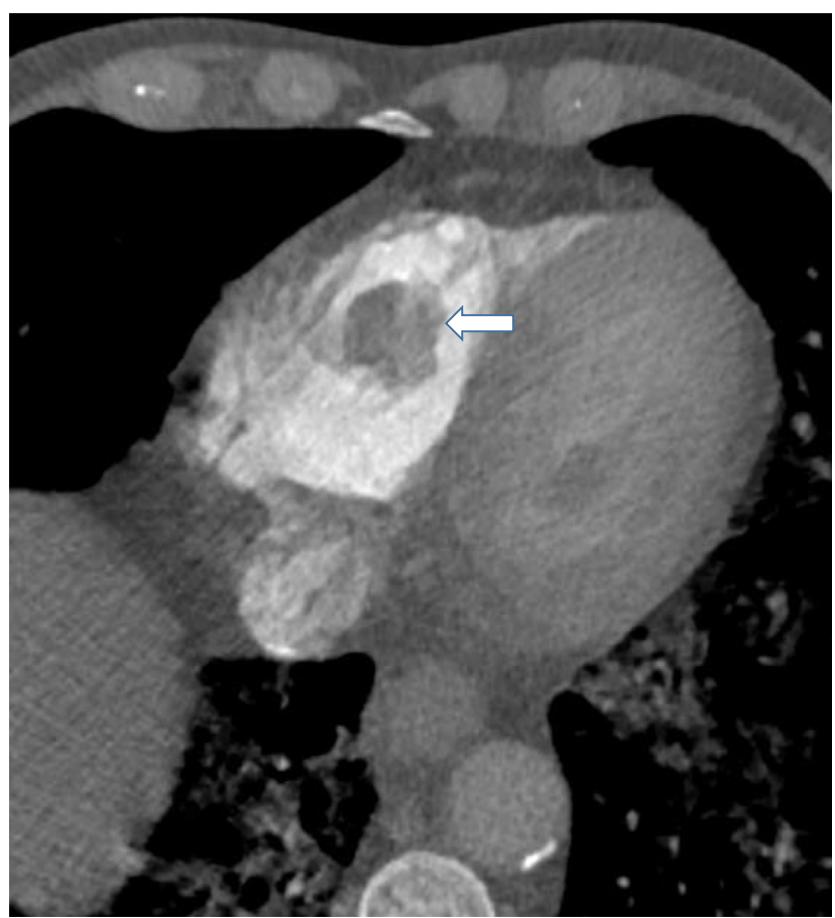

Fig. 3 A 62-year-old man who presented with shortness of breath, cough, and fever. CTA demonstrates bilateral pulmonary ground-glass opacities, bilateral lower lobe segmental pulmonary emboli (not shown), and right ventricular thrombus (white arrow). The latter finding was confirmed on transthoracic echocardiography

weakness, left facial paralysis, and decreased responsiveness. CT head noted the presence of an acute right parietal infarct and focal distal ascending aortic thrombus (Fig. 5a). As the diagnosis of acute stroke was made within the acceptable timeframe, thrombolysis with t-PA was given. His ICU course was complicated by hemodynamic instability, as well as rapid atrial fibrillation. On hospital day 12, he was noted to have absent pulses in the left arm and leg concerning for arterial thromboembolism. He was transferred to another hospital for vascular surgical evaluation of left lower extremity ischemia. Lower extremity arterial Doppler ultrasound revealed absent flow distal to the left popliteal artery (Fig. 5b), supportive of acute arterial thrombosis. Neurologic examination at this time noted the absence of corneal, gag, and oculocephalic reflexes. Patient went into cardiopulmonary arrest and died 2 days later.

\section{Case 6. Celiac artery thromboembolic complications}

Case 6 is a 61-year-old man with essential hypertension, type 2 diabetes mellitus, and hyperlipidemia admitted for COVID19 pneumonia after 5 days of fever, cough, and dyspnea. With progressive hypoxemic respiratory failure, the patient required mechanical ventilation on hospital day 5. Patient was subsequently diagnosed with ST elevation myocardial infarction and treated conservatively with intravenous heparin. His course was further complicated by gastrointestinal bleeding, and subsequent CTA of the chest, abdomen, and pelvis identified thrombi within his aorta, celiac artery (Fig. 6), and left internal jugular vein. Due to poor prognosis, patient and family decided to not escalate level of care. The patient expired 2 weeks after admission.

\section{Discussion}

Coronavirus disease 2019 (COVID-19) is a viral respiratory illness first reported in December 2019 in Wuhan, China, that has since spread rapidly around the world. As of October 27, 2020 , there was a cumulative total of 43.6 million cases of COVID-19 globally, including 1.16 million deaths [2]. COVID-19 is caused by the severe acute respiratory syndrome coronavirus 2 (SARS-CoV-2) which spreads from human-tohuman transmission, primarily from respiratory droplets, and potentially from aerosolized particles and contaminated surfaces [3]. The virus is a single-strand RNA beta-coronavirus that is believed to enter cells by binding the angiotensin

Fig. 4 A 54-year-old man with 3 days of right flank pain and negative urinalysis who underwent abdominal CT. a Axial CT image demonstrates right lower lobe segmental pulmonary embolus (white arrow). b Coronal CT image shows thrombosed posterior tributaries of the right renal vein (white arrow) and renal infarction (white left bracket)
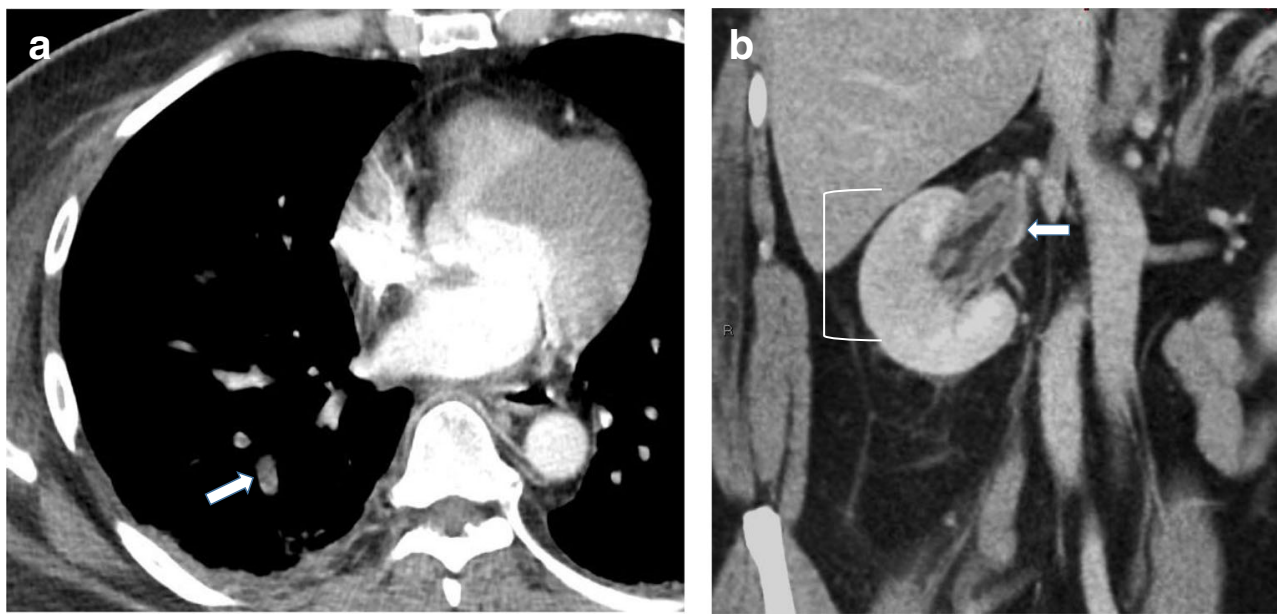

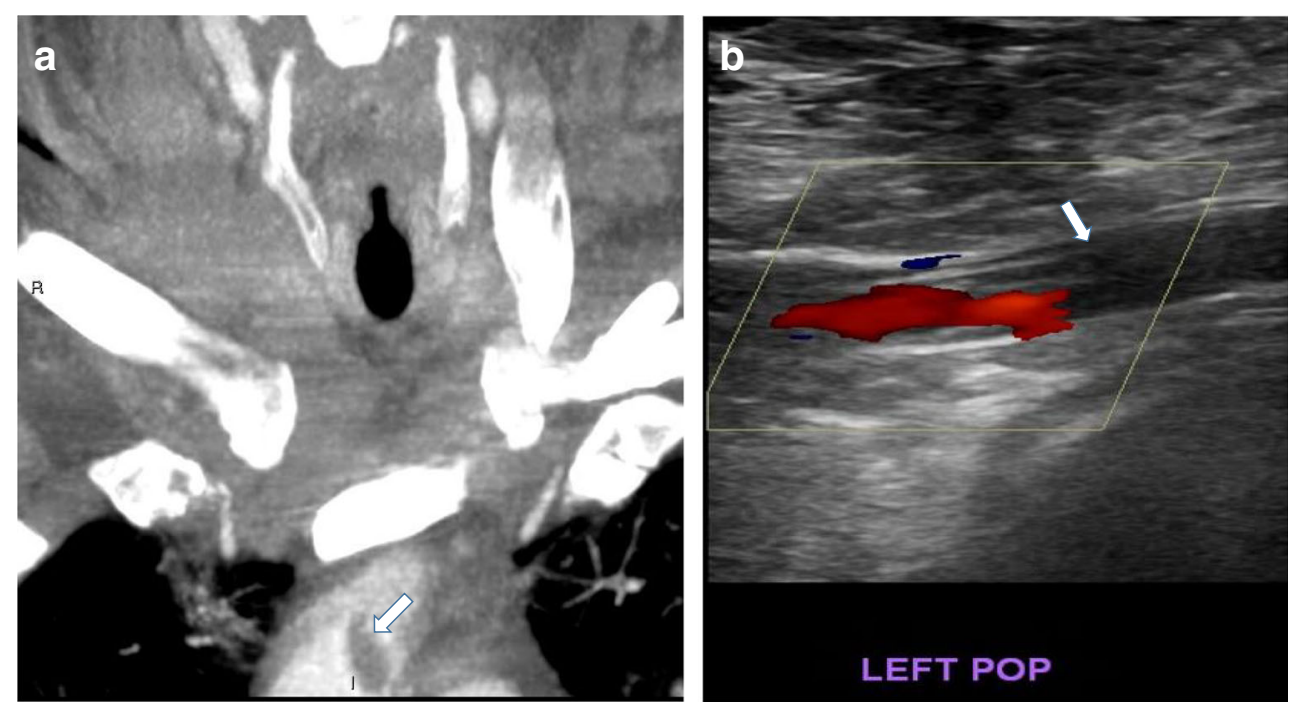

Fig. 5 A 50-year-old man admitted for COVID-19-associated pneumonia who developed sudden-onset left-sided weakness, left facial paralysis, and decreased responsiveness. After subsequent head CT revealed right parietal infarct, patient underwent head and neck CTA. a Coronal CTA image shows focal partially adherent distal aortic ascending thrombus (white arrow). b During admission, patient underwent lower extremity arterial Doppler ultrasound for a sudden onset of cold left foot. Color Doppler image demonstrates absent flow distal to the left popliteal artery (white arrow)

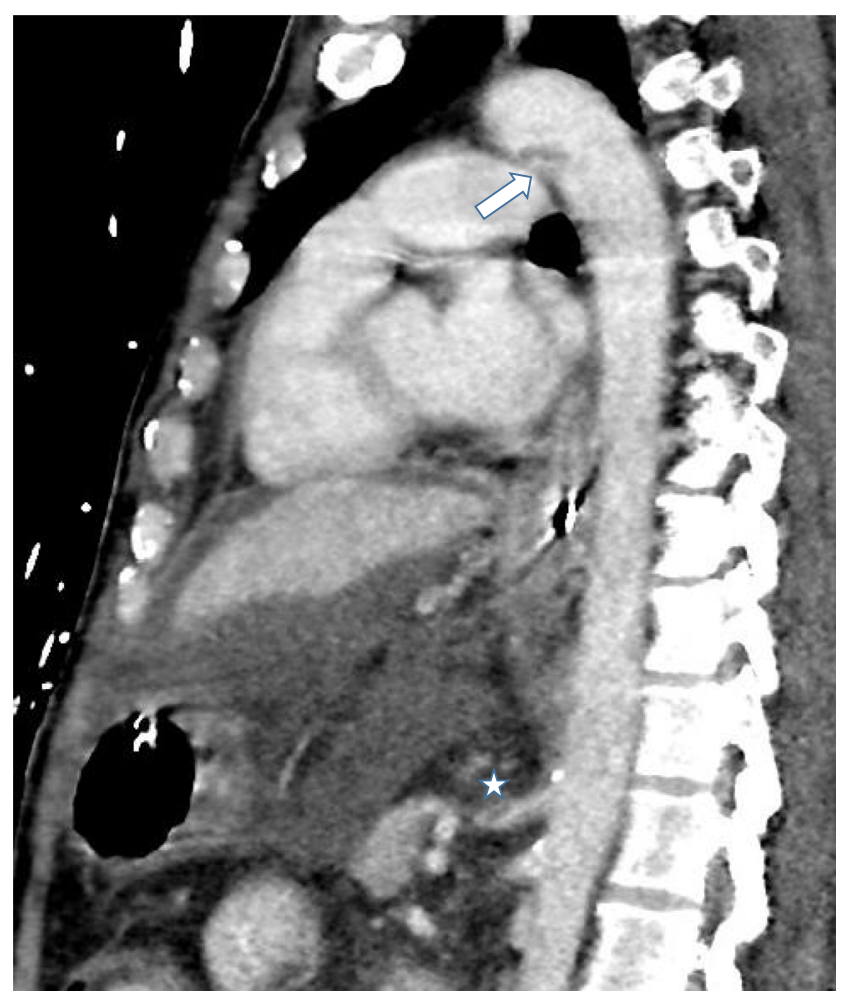

Fig. 6 A 61-year-old man with essential hypertension, type 2 diabetes mellitus, and hyperlipidemia admitted for COVID-19 pneumonia after 5 days of fever, cough, and dyspnea. His hospital course was complicated by ST elevation myocardial infarction, which was managed conservatively with parenteral anticoagulation and antiplatelet therapy. CT angiography of the chest, abdomen, and pelvis performed to assess rectal bleeding noted acute thrombi in his aortic arch (arrow), celiac artery (star), and left internal jugular vein (not shown) converting enzyme 2 (ACE2), found in lung alveolar cells, cardiac myocytes, and vascular endothelium [4]. The clinical manifestations include fever, cough, headache, back pain, abdominal pain, and anosmia [5]. As noted from early observational studies, risk factors for complications of COVID-19 include older age ( $>65$ years), cardiovascular disease, chronic lung disease, hypertension, diabetes, and obesity [6]. Initial clinical reports of COVID-19 patients focused on respiratory symptoms and chest CT imaging findings of bilateral groundglass opacities with peripheral and basal distribution [7, 8].

In moderate to severe cases of COVID-19, thrombotic complications have emerged in infected patients, as was seen with severe acute respiratory syndrome coronavirus 1 (SARSCoV-1) in 2003 [9]. There is currently limited data on thrombotic risk. Klok et al. found that $31 \%$ of ICU patients with COVID-19 infections at their hospital in the Netherlands had thrombotic complications, including pulmonary embolism or venous thromboembolism in $27 \%$ and arterial thrombotic complications in $3.7 \%$ [10]. In comparison, within a large New York City health system, $29.4 \%$ of ICU patients with COVID-19 had a thrombotic event (13.6\% venous and $18.6 \%$ arterial), whereas $11.5 \%$ of non-ICU patients had events (3.6\% venous and $8.4 \%$ arterial) [11]. A study of 143 hospitalized patients with COVID-19 in Wuhan, China, found that $46 \%$ developed lower extremity deep venous thrombosis [12], higher compared to a study by Umapathi et al. that found approximately $30 \%$ of critically ill patients with SARS-CoV had suffered venous thromboembolism [13]. There is scant data investigating thrombotic complications in patients infected with MERS-CoV. 
Thrombosis in both the arterial and venous systems is thought to arise from excessive inflammation, platelet activation, endothelial dysfunction, and stasis [14]. Combination of underlying medical conditions, hospitalization, bedridden status, and COVID-19 infection likely contributed to increased hypercoagulopathy in these patients. There is a strong association between D-dimer levels, disease progression, and prognosis $[15,16]$.

Thromboembolic events include pulmonary embolism, which may exacerbate respiratory failure and hypoxemia. Arterial thrombotic events include cerebrovascular accidents, end-organ ischemia to systemic organs and limb ischemia [17]. Our case series illustrates the spectrum of thromboembolic events directly affecting the cardiovascular, neurovascular, pulmonary, renal, and gastrointestinal systems. These examples highlight the importance for radiologists to identify the potentially farreaching coagulopathic effects caused by COVID-19. The radiologic evaluation of the sick COVID-19 patient should pay special attention the pulmonary arterial vascular system, as concomitant pulmonary emboli may explain persistent hypoxemia in the setting of oxygen supplementation and steroid use. Focal nonadherent thrombi may be seen in the right and left ventricles, aorta, iliac vessels, and branch vessels. Coronary arterial thrombosis is proposed to be a mechanism for sudden cardiac arrhythmias and death. EKG-gated image acquisition would be necessary for optimal CT coronary vessel evaluation.

Further studies are needed to identify risk factors for systemic thrombosis, including genetic factors and concomitant medical conditions. Larger sample sizes are necessary to quantify the incidence of thromboembolic complications in mild, moderate, and severe cases of COVID19. The identification of useful serum markers to assess patients at risk for thromboembolic events, including inflammatory markers such as D-dimer and fibrinogen levels, may help stratify coagulopathic risks in COVID19 patients and identify specific cohorts who may derive significant clinical benefits from anticoagulation therapy [18-20]. Experts agree that thrombotic risk is sufficient to recommend venous thromboembolism (VTE) prophylaxis in severe cases of COVID-19 as long as there is no contraindication.

Acknowledgments We thank the Northwell COVID-19 Research Consortium for fostering a supportive environment for clinical research and collaboration during time of crisis. We thank our colleagues Reena Malhotra, Piyush Banker, Paul Lee, and Priya Shah for their clinical contributions.

\section{Compliance with ethical standards}

Conflict of interest The authors declare that they have no conflicts of interest.

\section{References}

1. Belluck P (2020) Younger adults make up big portion of coronavirus hospitalizations in U.S. New York Times March 18, 2020: https://www.nytimes.com/2020/03/18/health/coronavirus-youngpeople.html?searchResultPosition=1

2. Johns Hopkins Center for Systems Science and Engineering. https://coronavirus.jhu.edu/map-faq

3. Van Doremalen N, Bushmaker T, Morris DH, Holbook MG, Gamble A, Williamson BN, Tamin A, Harcourt JL, Thornburg NJ, Gerber SI, Lloyd-Smith JO, de Wit E, Muster VJ (2020) Aerosol and surface stability of SARS-CoV-2 as compared with SARS-CoV-1. NEJM 382:15641567-15641567. https://doi.org/ 10.1056/NEJMc2004973

4. Oudkerk M, Buller HR, Kuijpers D, van Es N, Oudkerk SF, McLoud TC, Gommers D, van Dissel J (2020) Diagnosis, prevention, and treatment of thromboembolic complications in COVID19: report of the National Institute for the Public Health of the Netherlands Published Online: Apr 232020 https://doi.org/10. 1148/radiol.2020201629

5. Huang C, Wang Y, Li X, Ren L, Zhao J, Hu Y (2020) Clinical features of patients infected with 2019 novel coronavirus in Wuhan, China. Lancet 395:497-507

6. Richardson S, Hirsch JS, Narasimhan M (2020) Presenting characteristics, comorbidities, and outcomes among 5700 patients hospitalized with COVID-19 in the New York City area. JAMA Published online April 22, 2020. doi:https://doi.org/10.1001/jama. 2020.6775

7. Han R, Huang L, Jiang H, Dong J (2020) Early clinical and CT manifestations of coronavirus disease 2019 (COVID-19) pneumonia. AJR: 1-6. https://doi.org/10.2214/AJR.20.22961

8. Bernheim A, Mei X, Huang M, Yang Y, Fayad Z, Zhang N, Diao $\mathrm{K}$, Lin B, Zhu Xiqi Z, Li K et al (2020) Chest CT findings in coronavirus disease-19 (COVID-10): relationship to duration of infection. Radiology published Online Ahead of print: Feb 20 $2020 \mathrm{https} / / /$ doi.org/10.1148/radiol.2020200463

9. Giannis D, Ziogas IA, Gianni P (2020) Coagulation disorders in coronavirus infected patients: COVID-19, SARS-CoV-1, MERS$\mathrm{CoV}$ and lessons from the past. J Clin Virol $127 \mathrm{https} / /$ doi.org/10. 1016/j.jcv.2020.104362

10. Klok FA, Kruip MJHA, van der Meer NJM, Arbous MS, Gommers DAMPJ, Kant KM, Kaptein FHJ, van Paassen J, Stals MAM, Huisman MV, Endeman H (2020) Incidence of thrombotic complications in critically ill ICU patients with COVID-19. Thromb Res Apr 10; S0049-3848(20)30120-1. doi: 10.1016/ j.thromres.2020.04.013.

11. Bilaloglu S, Aphinyanaphongs Y Jones S, Iturrate E, Hochman J, Berger JS (2020) Thrombosis in hospitalized patients with COVID19 in a New York City Health System. JAMA Published online July 20, 2020. doi:https://doi.org/10.1001/jama.2020.13372

12. Zhang L, Zhang D, Jiang C, Mei H, Wang J, Zhang C et al (2020) Deep vein thrombosis in hospitalized patients with COVID-19 in Wuhan, China: Prevalence, risk factors, and outcome. Circulation. 2020; 142:114-128. doi:10.1161/CIRCULATIONAHA.120.046702

13. Umapathi T, Kor AC, Venketasubramian N, Lim CCT, Pang BC, Yeo TT et al (2004) Large artery ischaemic stroke in severe acute respiratory syndrome (SARS). J Neurol 251:1227-1231

14. Bikdeli B, Madhavan MV, Jimenez D, Chuich R, Dreyfus I, Driggin E et al. (2020) COVID-19 and thrombotic or thromboembolic disease: implications for prevention, antithrombotic therapy, and follow up. J Am Coll Cardiol Apr 15. doi: 10.1016/ j.jacc.2020.04.031.

15. Zhang L, Yan X, Fan Q, Liu H, Liu X, Liu Z, Zhang Z (2020) Ddimer levels on admission to predict in-hospital mortality in patients with Covid-19. Apr 19. doi: 10.1111/jth.14859. 
16. Lippi G, Favaloro (2020) D-dimer is associated with severity of coronavirus disease 2019: a pooled analysis. Thrombosis and Hemostasis 120(5):876-878. https://doi.org/10.1055/s-00401709650

17. Li Y, Wang M, Zhou Y, Chang J, Xian Y, et al (2020) Acute cerebrovascular disease following COVID-19: a single center, retrospective, observational study. Lancet: https://ssrn.com/abstract= 3550025, March 3, 2020.

18. Thachil J, Tang N, Gando Satoshi G, Falanga A, Cattaneo M et al (2020) ISTH interim guidance on recognition and management of coagulopathy in COVID-19. J Thromb Haemost. 18(25 March 2020):1023-1026. https://doi.org/10.1111/jth.14810
19. Tang N, Bai H, Chen X, Gong J, Li D, Sun Z (2020) Anticoagulant treatment is associated with decreased mortality in severe coronavirus disease 2019 patients with coagulopathy. J Thromb Haemost 18(5):1094-1099. https://doi.org/10.1111/jth.14817

20. Paranjpe I, Fuster V, Lala A, Russak A, Glicksberg BS, Levin MA, Charney AW, Narula J, Fayad ZA, et al (2020) J Am Coll Cardiolog. 2020 May 5;S0735-1097(20)35218-9. doi: 10.1016/ j.jacc.2020.05.001.

Publisher's note Springer Nature remains neutral with regard to jurisdictional claims in published maps and institutional affiliations. 